\title{
Accurate Finite Element Model of Equiaxed-grain Engineering Material for Ultrasonic Inspection
}

\author{
Bo Xiao ${ }^{I}$, Richard O'Leary ${ }^{I}$, Anthony Gachagan ${ }^{1}$ \\ ${ }^{1}$ Centre for Ultrasonic Engineering, \\ University of Strathclyde, \\ Glasgow, UK \\ Corresponding email: a.gachagan@strath.ac.uk
}

\author{
Wenqi Li $^{2}$, Timoth Burnett ${ }^{3}$ \\ ${ }^{2}$ Faculty of Engineering, University of Nottingham, \\ Nottingham, UK \\ ${ }^{3}$ School of Material, University of Manchester, \\ Manchester, UK
}

\begin{abstract}
Finite element (FE) simulation of grained material with equiaxed grain distribution is of interest for the virtual prototyping of array structures and the assessment of signal processing algorithms. Construction of such models can be computationally intensive due to the large number of crystallographic orientations required to represent the material. This paper concentrates on analysis and processing of orientation data in order to establish a computationally efficient 2D FE model whilst maintaining appropriate accuracy of the grained structure. Two approaches for orientation processing are proposed and their performances are compared. Parametric studies show that the trade-off between computational overhead and model accuracy will reach the optimal point when Euler space is segmented with a bin size of 15 degree per Euler phase. A transducer array is then incorporated into the $F E$ model to generate B-scan image of the material. The image is compared with experimental equivalent for $\mathrm{FE}$ model validation purpose. The minor difference of images proves that the constructed FE model is accurate, highlighting the potential of the proposed methods for application on other equiaxed-grain materials.
\end{abstract}

Keywords-Material characterization, equiaxed-grain material, accurate modelling

\section{INTRODUCTION}

Ultrasonic inspection of polycrystalline engineering materials currently represents a significant challenge. Beam path is skewed and distorted due to the anisotropy and inhomogeneity of the medium, giving rise to phase aberration resulting in inaccuracy in defect detection and characterization [1]. Recently, researchers have tried to identify phase aberration and modify the delay law by modelling analysis [2]. The modified delay law can then be used as a practical approach to improve focusing in the load. Establishment of an effective model requires grain orientation information and a high degree of accuracy from the model is required.

Electron backscatter diffraction (EBSD) is a well-known technique to determine the crystallographic orientations of polycrystalline materials [3]. EBSD is implemented over relatively small areas within a sample - a few $\mathrm{mm}^{2}$ being typical. Alternatively, a wide area technique known as Spatially Resolved Acoustic Spectroscopy (SRAS) has been developed recently [4]. SRAS is implemented in an optical scanning acoustic microscope where a wide band laser pulse passes through a mask with fixed fringe spacing, illuminating a region of the sample and exciting surface acoustic waves
(SAW). The frequency with maximum amplitude is then used to determine SAW velocity. By rotating the sample, SAW velocities at different angles are obtained, which are then converted to crystallographic orientations using a method described in [4]

The orientation information can be input into either a (semi-) analytical model or a finite element (FE) model. However, for polycrystalline materials, (semi-) analytical models can rapidly reach its limit in terms of computational capacity and accuracy due to the large number of grains and grain boundaries present along the ultrasound wave propagation path [5]. In contrast, the presence of a multitude of grain boundaries typically has little or no additional impact on the computational cost for a FE model. Therefore, as material complexity increases, FE simulation becomes more advantageous.

For polycrystalline materials, crystallographic orientations should be quantitatively reduced prior to construction of the FE model. Otherwise, the large number of orientations required within the model will result in a significant computational overhead in construction/pre-processing phase the model. Most existing relevant research focuses on constructing FE model of columnar-grain materials such as austenitic welds, in which the non-dominant crystallographic orientations are unified with the nearest identified dominant orientation in a process termed orientation unification. Since there are usually only several (<10) dominant orientations present in the material, a computationally efficient and accurate FE model can be readily established [2]. In this context model accuracy relates to the minimizing the effects of the orientation processing such that the model as closely as possible represents the orientation measured in the sample. However, equiaxed-grain materials exhibit microscopically randomly oriented grains whose crystallographic orientations have an even distribution in the orientation space. As no dominant orientation exists, new orientation processing methods instead of orientation unification are desired.

This paper will focus on creating a computationally efficient FE model of an equiaxed-grain Inconel 617 sample whilst maintaining model accuracy. Orientations are preprocessed by two proposed methods termed Euler Binning (EB) and Grain Unification with Euler Binning (GUEB). The relative performance of the two methods is then compared. Validation is carried out by comparison of experimental and FE derived B-scan images of the sample. 


\section{METHODOLOGY}

The Inconel alloy 617 sample provided by E.ON (Ratcliffeon-Soar, UK) is part of a cylindrical casting with $50 \mathrm{~mm}$ wall thickness. This alloy is an attractive construction material component in applications such as aerospace, power plants and gas turbines due to an exceptional combination of oxidation resistance and high-temperature modulus [6]. Moreover, it is a representative equiaxed-grain material with cubic crystal system.

\section{A. Material characterization}

SRAS measurement on the sample with a resolution size of $25 * 50 \mu \mathrm{m}$ was undertaken by the University of Nottingham. The measured velocities were averaged every four rows to smooth out the instrumentation noise, resulting final orientation resolution of $100 * 50 \mu \mathrm{m}$. Assume a $2.25 \mathrm{MHz}$ transducer array emitting a longitudinal wave with a wavelength of $\sim 2.5 \mathrm{~mm}$ is employed to inspection the material. Since the resolution sizes are smaller than wavelength so that they are considered as sufficient to accurately model wave propagation [2].

In order to make comparison to SRAS, the subsections of the sample were then characterized using EBSD measurement with resolution of $10^{*} 10 \mu \mathrm{m}$ and no averaging applied. Since orientation resolutions of two datasets are significantly different, fair comparison of orientations are not achievable. Instead, Fig.1 shows the comparisons of one subsection (1.92 $* 1.71 \mathrm{~mm}$ ) between one SAW velocity map measured by SRAS in Fig.1 (a) and inverse pole figure map in y direction of sample frame generated by EBSD in Fig.1 (b). A certain color corresponds to a certain velocity for SRAS and to a certain orientation viewed from y direction for EBSD. The two maps, especially the grain boundaries, are in good agreement.

\section{B. FE modelling}

Generally, crystallographic orientations are expressed as three Euler angles $(\varphi 1, \varphi, \varphi 2)$ by rotations of which in a specific order the sample frame will coincide with local crystal frame. The complete Euler angles are written into a separate material file in which materials are defined using the Euler angles and the ensemble elastic constants (i.e. average over a large number of grains). The material file is then read into the FE modelling package suite PZFlex (Weidlinger Associates Ltd., CA) and material properties are assigned to corresponding elements before a model starts to run in PZFlex.

Orientation distribution function (ODF) of the equiaxed Inconel 617 sample is displayed as Fig. 2 where each subplot is displayed with 10 degree range of $\varphi_{2}$ and full range of $\varphi$ $\left(0^{\circ} \sim 360^{\circ}\right)$ and $\varphi_{1}\left(0^{\circ} \sim 90^{\circ}\right)$. Ranges of $\varphi$ and $\varphi 2$ are reduced to $0^{\circ} \sim 90^{\circ}$ by applying symmetry properties of cubic system. Orientations are observed to evenly distribute in the orientation space, therefore, reduction of orientations number is desired to decrease computation overhead of material file read-in and properties assignment.

It is an intuitive thought to segment the orientation space into a reasonable number of bins and then unify each bin by setting all orientations to the dominant one. This method is termed as Euler Binning (EB). Assume that the Rayleigh scattering scheme is dominant [7], ultrasound wave is sensitive to grain with minimum size $1 / 10$ of wavelength $(\sim 250 \mu \mathrm{m}$ for

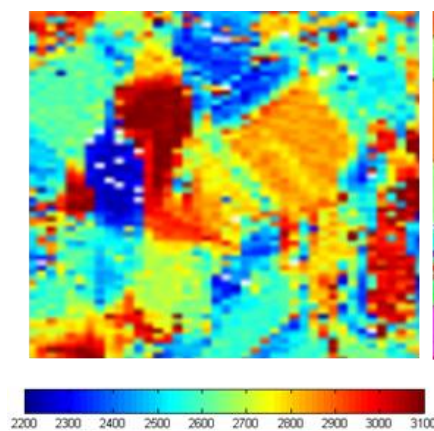

(a)

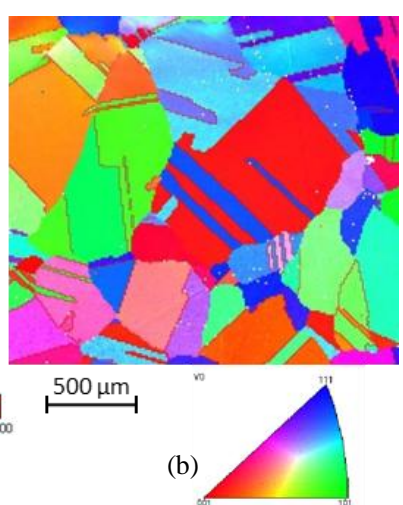

Fig.1. Comparisons of SRAS (a) and EBSD (b) data of a same section of the sample. A certain color corresponds to a certain velocity for SRAS and to a certain orientation viewed from y direction for EBSD.

2.25 MHz). After defining of grain boundaries using certain misorientation angles, significant grains, i.e., grains whose sizes are comparable to or larger than 1/10 wavelength, are expected to remain and their Euler angles are required to be less altered after orientation processing. In EB method, a significant grain is possibly segmented into two or more bins, resulting in loss of significant grain structures. Meanwhile, two or more insignificant grains are possibly unified as a single significant grain, giving rise to grain artefacts. Hence, another method which spatially identifies significant grains and then unifies each grain followed by segmentation of orientation space and bin unification is proposed, termed as Grains Unification with Euler Binning (GUEB). This method is able to avoid grains loss and more equitably suppress grain artefacts by optimizing parameters.

A trade-off between computation overhead and grain accuracy is established. As bins size increase, the computation overhead will decrease but model accuracy, more importantly, accuracy of significant grains will decrease due to a larger degree of approximation during bin unification stage. The optimal bin size for the trade-off will be studied later.

\section{Experimental validation}

A 1D linear transducer array was also simulated in PZFlex along with the optimized material model. Sequentially, we stimulated each element of the array while all elements were used as receivers, thus Full Matrix Captured (FMC) data were collected and used to form B-scan image using Total Focusing Method (TFM) [8]. The B-scan image will be compared with experimental equivalence in order to validate the material model in next section.

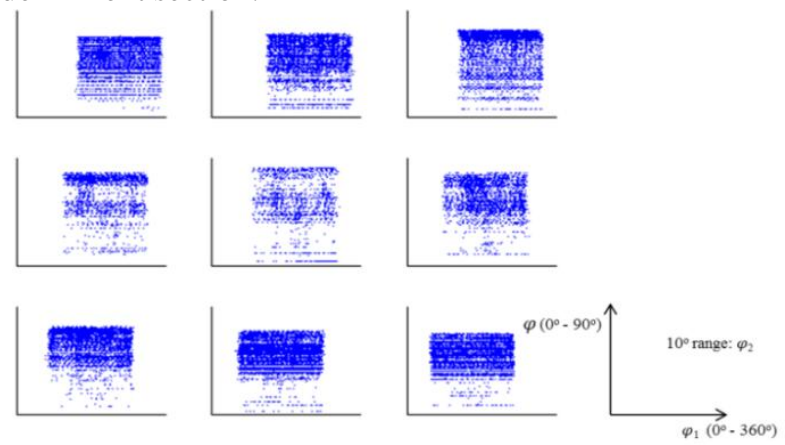

Fig. 2. ODF of the Inconel 617 sample. Each subplot is displayed with 10 degree range of $\varphi 2$ and full range of $\varphi\left(0^{\circ} \sim 360^{\circ}\right)$ and $\varphi 1\left(0^{\circ} \sim 90^{\circ}\right)$. 
(a)

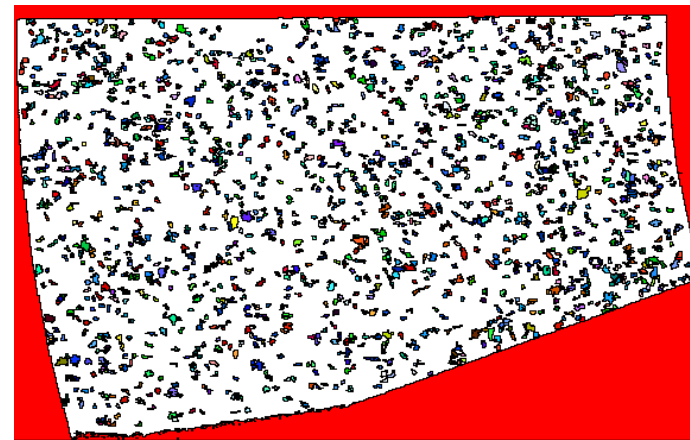

(b)

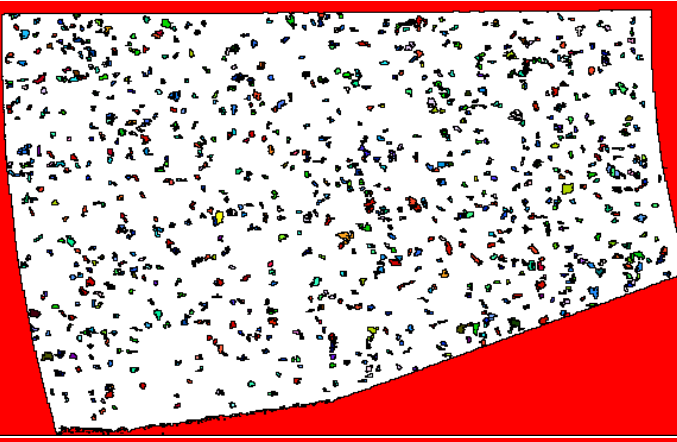

(c)

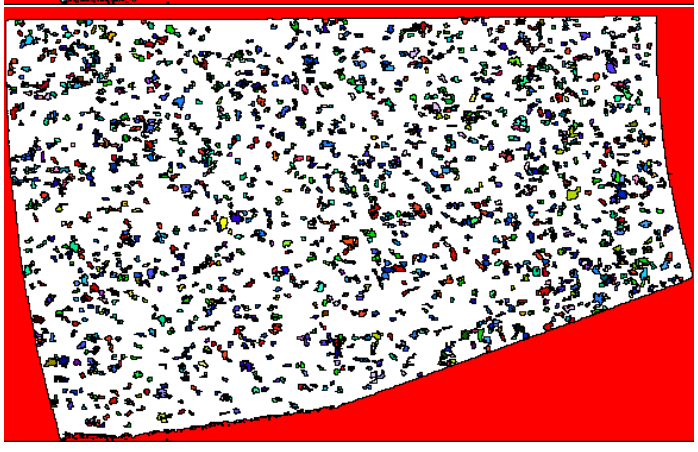

Fig. 3. Distribution of significant grains for: (a) original data; (b) EB processing; (c) GUEB processing. In all figures, red region is the background; coloured components of the material are significant grains; white components are insignificant grains.

\section{RESULTS}

\section{A. Model accuracy}

Fig. 3 (a) displays the distribution of significant grains within the material map in the case of bin size of 15 degree per Euler phase, misorientation angle of $10^{\circ}$ per phase, and inspection frequency of $2.25 \mathrm{MHz}$. Fig. 3 (b) and (c) show significant grains distribution for EB and GUEB processing, respectively. In all figures, the red region surrounding the keystone shaped sample is the background; colored components of the sample are significant grains; white components are insignificant grains. A large loss of significant grains is observed in the EB processing result; in contrast, EBGU retains the significant grains and introduces a low level of grain artefacts. These figures can also be expressed as binary maps in which element of significant grains are set as 1 and elements of insignificant grains are set 0 . The error in the significant grains distribution introduced by EB and GUEB processing can be quantified as correlation coefficient between original binary map and processed binary map. The correlation coefficient $\mathrm{C}$ is formulated as

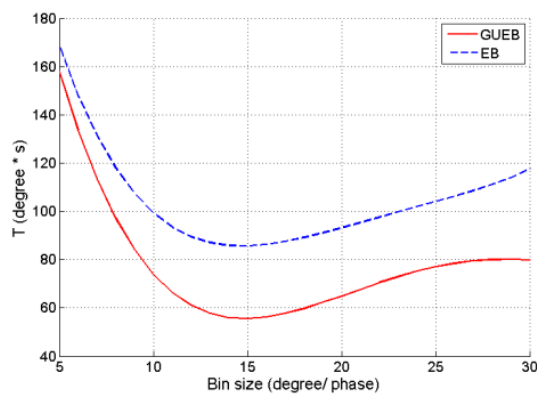

Fig. 4. The trade-off $\mathrm{T}$ changes with bin size

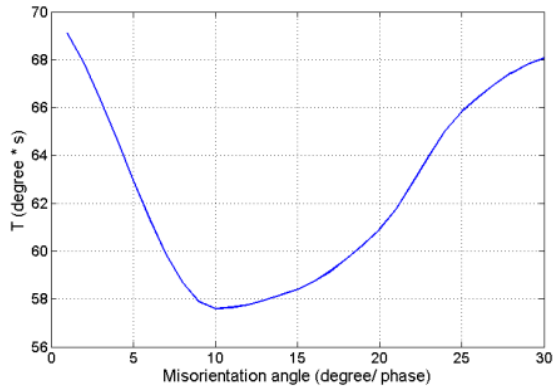

Fig. 5. The trade-off T changes with misorientation angle for GUEB approach

$$
C=\frac{\sum_{x=1}^{m} \sum_{y=1}^{n}\left(B_{0}(i, j)-\overline{B_{0}}\right)\left(B_{a}(i, j)-\overline{B_{a}}\right)}{\sqrt{\left(\sum_{x=1}^{m} \sum_{y=1}^{n}\left(B_{0}(i, j)-\overline{B_{0}}\right)^{2}\right)\left(\sum_{x=1}^{m} \sum_{y=1}^{n}\left(B_{a}(i, j)-\overline{B_{a}}\right)^{2}\right)}}
$$

where $m$ and $n$ are numbers of elements in lateral $(x)$ and axial $(y)$ and directions, respectively; $B_{o}$ and $B_{a}$ are binary values for original and processed binary map, respectively. Assume that the original significant grains have a total number of $N$ elements, and that original Euler angles and the processed ones are expressed as $E_{o}$ and $E_{a}$, respectively. Mean square error (MSE) of Euler angles of the significant grains can be formulated as

$$
M S E=\sqrt{\frac{\sum_{i=1}^{N} \sum_{k=1}^{3}\left(E_{0}(i, k)-E_{a}(i, k)\right)^{2}}{3 * N}}
$$

We then define

$$
T=M S E * \text { Time } / C
$$

where Time is the material read-in time. Note that Time values at different bin sizes were measured at the same computer/ software conditions. The trade-off between significant grains accuracy and computation overhead will reach the optimal point when $T$ has its minimum value. Fig. 4 shows the how the $T$ changes with bin size for both EB and GUEB processing approaches. $T$ is observed to reach the minimum value at bin size of 15 degree per Euler phase for both EB and GUEB approaches. GUEB approach has lower $T$ values for a range of bin size than EB approach. Particularly, $T$ value of EB is around $47 \%$ higher than the GUEB counterpart $\left(M S E / C \sim 4^{\circ}\right)$ at bin size 15 degree.

The number of significant grains needed to be retained after processing depends on the inspection frequency and misorientation angle used to define the grain boundaries. The appropriate inspection frequency is dependent on orientation data resolution and averaged grain size, namely, it is already 
(a)

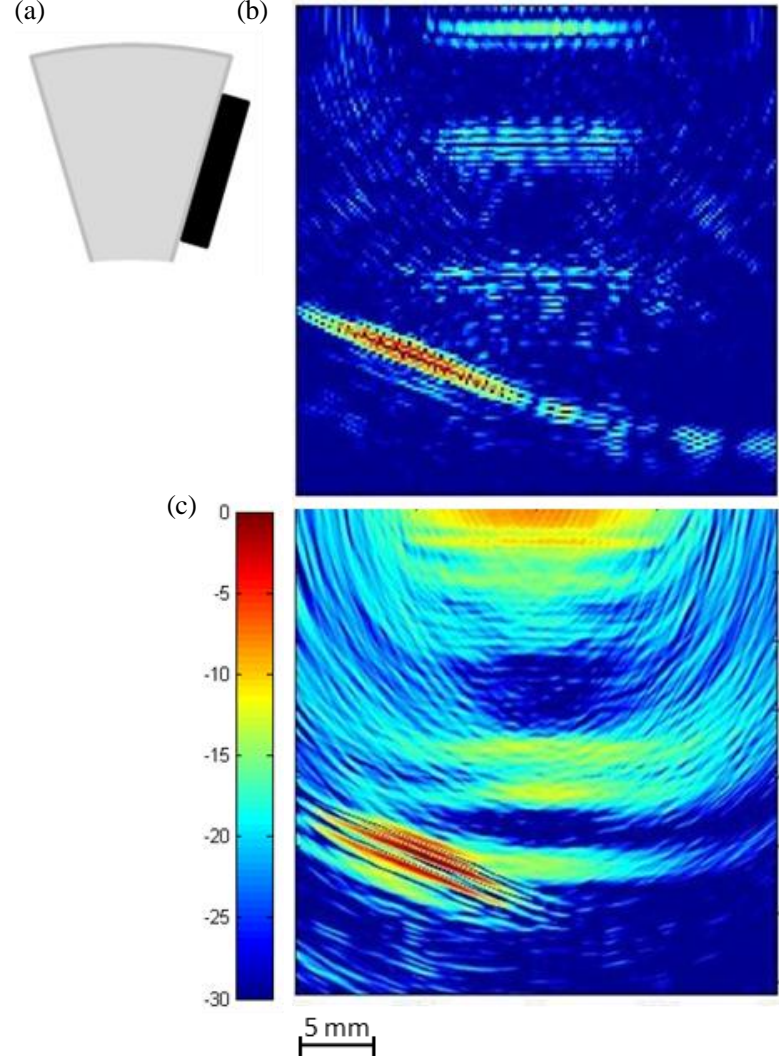

Fig. 6. (a) array location for both simulation and experiment; (b) TFM image of FE simulation; (c) experimental TFM image. Both TFM figures are shown with dynamic range of $-30 \mathrm{~dB}$.

determined during material characterization stage. Misorientation angle, however, can be altered to study how it affects the performances of orientation processing approaches. As misorientation angle increases, spatially adjacent insignificant grains will possibly merge as a significant grain whereas adjacent significant grains will possibly merge as a single significant grain. Fig. 5 shows the $T$ value reaches its minimum at misorientation angle of 10 per phase in the case of bin size of 15 degree per phase and $2.25 \mathrm{MHz}$ inspection frequency for GUEB approach. $T$ values have a range of $17 \%$ variation for other chosen misorientation angles compared with the minimum.

\section{B. Experimental validation}

Subsequently, 15 degree per Euler phase was chosen to segment orientation space into 356 bins with unification of significant grains where misorientation angle was chosen as 10 degree per phase. A 1D transducer array 32 elements, 2.25 $\mathrm{MHz}, 1 \mathrm{~mm}$ pitch size was simulated in the FE model on the flat side of the Inconel as shown in Fig. 6(a). FMC datasets were collected and used to form TFM image. In experimental stage, the array was placed on the same location as simulation and data acquisition and image formation were also carried out. Therefore, the model can be validated by comparing its TFM image with experimental TFM image. For the reason of fair comparison, Gaussian noise was added into the FE model to approximate the effect of microstructure variations across experimental array. Fig. 6 (b) and (c) illustrates that the two TFM images are in agreement with each other, especially the locations of back wall and strong speckle regions. Note that each TFM image is normalized and then logarithmically compressed. Slight difference of array parameters and model inaccuracy are responsible to images discrepancies.

\section{CONCLUSION AND FUTURE WORK}

In this paper, we present two orientation processing approaches in order to create cost-effective FE model of material with equiaxed grain distribution. Parametric studies illustrate the trade-off between computation overhead and model accuracy reaches the optimal point for bin size of 15 degree per Euler phase and misorientation angle of 10 degree per Euler phase. The model is then validated by comparing its TFM image with experimental equivalent. Future works include two aspects. Firstly, the two proposed orientation processing approaches both are unable to completely prevent the introduction of grain artefacts. Extra correction step will be studied to maximize grain accuracy. Secondly, a side hole will be drilled on the material sample and also be inserted into the model at same location. The FE model will then be further validated in terms of effectiveness in improving practical focusing.

\section{ACKNOWLEDGMENT}

The authors acknowledge support from EPSRC (EP/I021027/1) Rolls Royce, Shell, National Nuclear Laboratories, AMEC, and Weidlinger Associates under the auspices of UK RCNDE. E.ON Technologies and Siemens AG are also acknowledged for providing the Inconel 617 sample discussed in this paper. Dr Ralf Hielscher's research group is greatly acknowledged for their MTEX open source software used for EBSD and SRAS data analysis.

\section{REFERENCES}

[1] M. O'Donnell and S. W. Flax, "Phase aberration measurements in medical ultrasound: human studies," Ultrason Imaging, vol. 10, pp. 1-11, 1988.

[2] C. Nageswaran and C. Carpentier, "Microstructural quantification, modeling and array ultrasonics to improve the inspection of austenitic welds," Insight, vol. 51, pp. 600-616, 2009.

[3] V. Randle and O. Engler, Introduction to Texture Analysis, Macrotexture, Microtexture, and Orientation Mapping. New York: Gordon and Breach, 2000.

[4] W. Li, S.D. Sharples, R. J. Smith, M. Clark, and M. G. Somekh, "Determination of crystallographic orientation of large grain metals with surface acoustic waves.," The Journal of the Acoustical Society of America, vol. 132, 2012.

[5] W. Lord, R. Ludwing, and Z. You, "Developments in ultrasonic modeling with finite element analysis," Journal of Nondestructive Evaluation, vol. 9, pp. 129-143, 1990.

[6] P. Ganesan, G. D. Smith, and D. H. Yates, "Performance of Inconel Alloy 617 in Actual and Simulated Gas Turbine Environments, " Material and manufacturing process, Vol. 10, 1995

[7] J. Krautkramer and H. Krautkramer, Ultrasonic Testing of Materials. New York: Springer-Verlag, 1990.

[8] C. Holmes, B.W. Drinkwater, and P.D. Wilcox, "Post-processing of the full matrix of ultrasonic transmit-receive array data for non-destructive evaluation," NDT\&E, vol. 38, pp. 701-711, 2005. 